\section{RABDOMIOSARCOMA PARATESTICULAR: A PROPÓSITO DE UN CASO EN EL ADULTO.}

\author{
Ángel Andréu G arcía, Enrique Herrero Polo, Jose \\ Miguel Alapont Alacreu, Roberto Botella \\ Almodóvar, Enrique Schiefenbusch M unné, José \\ Luis Brotons Márquez, Pilar G arcía Arranz ${ }^{1}$ y \\ Gonzalo Lamazares Cachá.
}

Servicio de Urología, y Servicio de Anatomía Patológica del Hospital G eneral de Elda. Elda. Alicante. España.

Resumen.- O BJETIVO : Presentar un caso de rabdomiosarcoma paratesticular derecho en un adulto joven de 21 años de edad.

MÉTO DO S: Tras realizar tratamiento con orquiectomía radical derecha vía inguinal, que se clasifica dentro del grupo clínico IA según el IRS - III (enfermedad localizada completamente resecada), se complementa con vincristina y actinomicina $-D$.

RESULTADOS/ CON CLUSIO N ES: Encontrándose libre de enfermedad a los 18 meses de la intervención quirúrgica. Destacando la importancia del tratamiento adyuvante en la evolución posterior de la enfermedad tumoral.

Palabras clave: Tumor paratesticular Rabdomiosarcoma.
Summary.- O BJEC TIVES: To report one case of right paratesticular rabdomyosarcoma in a 21 -year-old young adult.

M ETHO DS: After treatment with right radical orchiectomy through an inguinal incision it was classified as clinical group I A following the IRS-III (localized disease completely excised) and received adjuvant treatment with Vincristine and actinomicin D.

RESULTS/ CON CLUSION S: The patient is disease free 18 months after surgery. W e emphasize the importance of adjuvant treatment in the posterior evolution of the tumoral disease.

Keyw ords: Paratesticular tumor.

Rabdomyosarcoma.

\section{INTRODUCCIÓN.}

De las neoplasias derivadas de las estructuras del cordón espermático, las más frecuentes son los tumores paratesticulares.

La mayor parte de estos suelen ser benignos (Lipomas), los tumores malignos representan aproximadamente el $30 \%$, fundamentalmente sarcomas ( $90 \%$ de casos), (3) y son propios del adulto (7).

El Rabdomiosarcoma (R.M.S.) es el sarcoma de tejidos blandos mas frecuente en niñez y adolescencia representando el $15 \%$ de las neoplasias pediátricas, siendo este tipo de sarcoma poco frecuente en adultos (3-4).

De todos los R.M.S. solo el $7 \%$ se localiza a nivel paratesticular. A este nivel el tumor puede invadir el testículo, cubiertas adyacentes, epidídimo y restos del conducto peritoneo-vaginal. Extendiéndose por vía linfática a ganglios localizados en retroperitoneo, pudiéndose ver afectados ganglios paraaórticos y paracavos hasta en un $70 \%$ de los casos (6).

Describimos un caso de R.M.S. testicular en el adulto, así como su tratamiento actual que, combina cirugía radical y poliquimioterapia, significando una mejoría en la supervivencia de estos pacientes (superior al $80 \%$ a los 2 años). (6). 


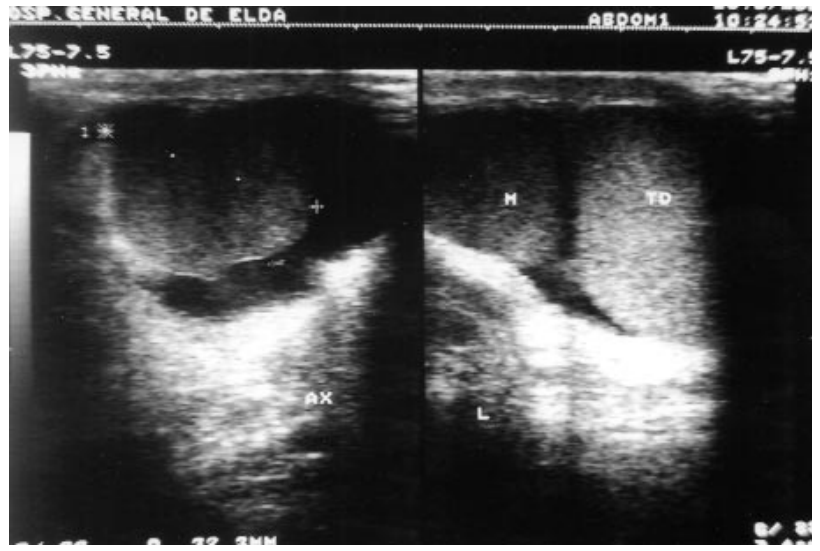

FIG URA 1. M asa extratesticular sólida de $3 \times 2.1 \times 2.5$ localizada a nivel de cabeza de epidídimo.

TABLA I. (2-5). CLASIFICACIÓ N C LIN ICA DEL R.M.S. SEG ÚN IN TERG RO UP RHABDO M IO SARCO M A STUDY III.

\begin{tabular}{|c|c|}
\hline Grupo & \multicolumn{1}{|c|}{ Definición } \\
\hline I & $\begin{array}{c}\text { Tumoración localizada y resecada por completo: } \\
\text { A) Confinada al músculo u órgano de origen } \\
\text { B) Infiltrada mas allá del origen }\end{array}$ \\
\hline II & $\begin{array}{c}\text { Resección completa con diseminación regional: } \\
\text { A) Resección total con enfermedad residual } \\
\text { microscopica } \\
\text { resecados sin enfermedad residual } \\
\text { C) Linfáticos regionales infiltrados y resecados } \\
\text { pero con diseminación microscópica } \\
\text { residual }\end{array}$ \\
\hline III & $\begin{array}{c}\text { A) Sólo se realiza biopsia } \\
\text { B) Resección parcial amplia de más del } 50 \% \\
\text { del volumen del tumor. }\end{array}$ \\
\hline diagnóstico. \\
\hline
\end{tabular}

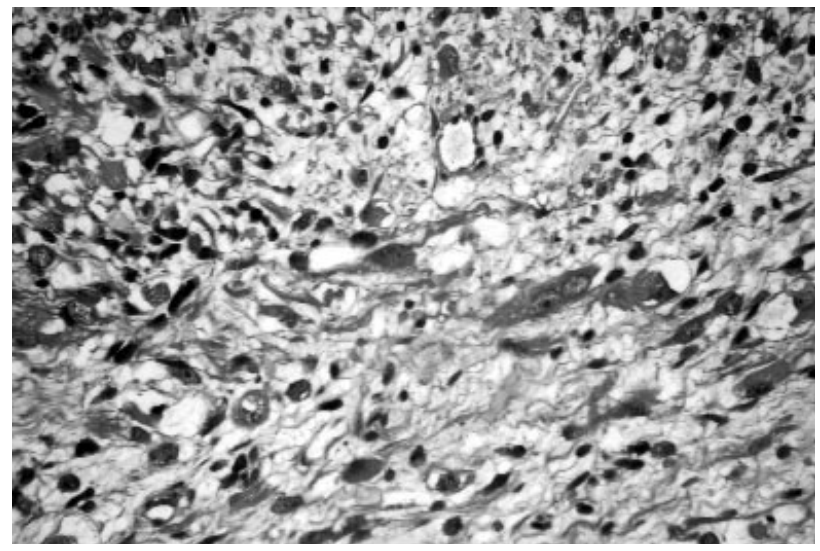

FIG URA 2. Tumoración mesenquial maligna de celularidad fusiforme con áreas mixoides y otras áreas con diferenciación rabdomioblásticas.

\section{CASO CÚNICO}

Paciente de 21 años de edad sin antecedentes personales de interés.

A cude a nuestro Hospital por presentar tumoración indolora en hemiescroto derecho, de aproximadamente 1 mes de evolución. En la exploración física se objetiva masa extratesticular de consistencia dura, independiente del testiculo. En la ecografia se objetiva masa extratesticular solida de $3 \times 2.1 \times 2.5$ (Figura 1) localizada a nivel de cabeza de epidídimo; se acompaña de hidrocele y es compatible con tumor sólido. La TAC abdominal y pelviana no demostró metastásis ni adenopatías. La radiografía simple de Tórax fue normal. Se realizaron marcadores tumorales Alfa FP y Beta HCG de valores normales.

Fue intervenido quirúrgicamente realizándose oquiectomía derecha por vía inguinal, con ligadura alta del cordón espermático, sin linfadenectomía retroperitoneal e implantación de prótesis testicular.

El estudio anatomopatológico lo etiquetó de rabdomiosarcoma embrionario que afecta a región paratesticular (vaginal testicular y epidídimo), estando respetados y libres de tumor ,cordón espermático, testículo y bordes quirúrgicos de la pieza (Figura 2).

Tras ser etiquetado como T2 N 0 M 0 según clasificación IN M y como estadio IA según el IRS (Tabla I) (2-5), pasa al Servicio de 0 ncología que lo somete a quimioterapia con 2 ciclos de vincristina y actinomicima D. Estando libre de tumor a los 18 meses de la intervención. 


\section{DISCUSIÓN}

Los sarcomas de cordón espermático son tumores relativamente poco frecuentes. Dentro de estos el R.M.S. paratesticular es mas frecuente en niños y adolescentes, siendo excepcional fuera de las dos primeras décadas de la vida. (6).

En general son tumores de gran agresividad biológica con tendencia a la recidiva local y posibilidad de metástasis a distancia, aproximadamente un tercio de los pacientes fallecen por enfermedad metastásica (8).

Se han descrito tres tipos histológicos: pleomórfico, embrionario y alveolar, (4) siendo el embrionario el que más frecuentemente se localiza en la zona paratesticular. El diagnóstico anatomopatológico, debe confirmarse en todos los casos con la realización de análisis inmunohistoquímicos.

Clínicamente se manifiesta como masas intraescrotal, firme, móvil e indolora, siendo la ecografía la que confirma el diagnóstico de masa sólida, descartándose otras posibles patologías como hernias o hidroceles primarios.

El tratamiento inicial recomendado para estos tumores es la realización de orquiectomía radical con ligadura alta de Cordón espermático, mas la utilización de otras medidas terapéuticas.

No debiéndose coger jamás la vía transescrotal por el gran riesgo de dejar tumor residual después de la exéresis de la masa (1), debiéndose evitar también la realización de biopsias transescrotales, ya que pueden dar lugar a extensión tumoral por vía linfática a ganglios lleofemorales y paraaórticos.

El Intergroup Rhabdiomiosarcoma Study preconiza para el estadio IA (Tabla I) la realización de orquiectomía radical y quimioterapia sistémica adyuvante, y para los estadios mas avanzados agregar Linfadenectomía retroperitoneal unilateral con radioterapia adyuvante en los casos de ganglios positivos.

La localización paratesticular es zona de buen pronostico dentro de los R.M.S. ya que además de sus características histológicas favorables, son tumores que suelen diagnosticarse precozmente dada su localización

En la actualidad el empleo combinado de cirugía radical junto con quimioterapia adyuvante, ha elevado la supervivencia en todos los estadios del $5 \%$ al $95 \%$ en los tres primeros años, y al $92 \%$ a los 5 años, en casi todas las series (6).

El pronóstico de estos pacientes ha mejorado con el tratamiento multidisciplinar así como con una cirugía precoz, aunque los resultados a largo plazo, están aún en estudio.

\section{BIBUOGRAFIA y LECTURAS \\ RECOMENDADAS (*lectura de interés y **lectura fundamental)}

1. DALl'IGNA, P.; BISOGNO, G.; FERRARI, A. y cols.: "Primary Transcrotal excision for paratesticular rhabdomyosarcoma is hemiescrotectomy really mandatory". Cancer, 97: 1998, 2003.

2. DE VRIES, J.D.: "Paratesticular rhabdomiosarcoma". World J. Urol., 13: 219, 1995.

3. J.R. SALADINI SFIER, E.; ALEXANDRO DASILVA, C.A.; CABEZAS CHECCI. y cols.: "Sarcoma paratesticular: A propósito de un caso con 19 años de evolución".

Actas Urol. Esp., 512, 1998.

*4. MARTIN-MARQUINA ASPIUNZA, A.; AROCENA GARCÍA-TAPIA, J.; SANZ PÉREZ, G. y cols.: "Rabdomiosarcoma paratesticular". Act. Urol. Esp., 22: 778, 1998.

5. RANEY, B.R. Jr.; TEFFT, M.; LAWRENCE, W. Jr. y cols.: "The Intergroup Rhabdiomyosarcoma Studies I and II. 1973-1983". Cancer, GO: 2337, 1987.

**6. MÉNDEZ GALLART, R.; GÓMEZ TELLADO, M.; MONTERO SÁNCHEZ, M. y Cols.: "Rabdiomiosarcoma paratesticular". Actas Urol. Esp., 23: 549, 1999. ALGABA, F.; MORENO, A.: "Uropatologia tumoral:

7. Correlación morfológica molecular y clínica". Pulso Ediciones Barcelona 1996.

CATTON, C.; N. CUMMINGS, B.J.; GORNASIER,

*8. V. y cols.: "Adults paratesticular sarcomas: a review of 21 cases”. J. Urol., 146: 342, 1991. 